\title{
Isolation of Neisseria meningitidis and Neisseria catarhalis from the genitourinary tract and anal canal
}

\author{
CAROLINE BLACKWELL, HUGH YOUNG, AND SHEILA S. R. BAIN* \\ From the Department of Bacteriology, University of Edinburgh, and the \\ University Department of Venereology, ${ }^{*}$ Edinburgh Royal Infirmary
}

SUMMARY During a 12-month period 285 isolates of Neisseria species, other than Neisseria gonorrhoeae, were cultured from patients attending the Department of Venereology, Royal Infirmary, Edinburgh. There were eight patients in whom genitourinary or rectal isolates of Neisseria meningitidis or Neisseria catarrhalis were found. Differences between data from our series and from previous reports are discussed with particular regard to sites routinely cultured in female patients. We also report in vitro inhibition of $N$. gonorrhoeae by an isolate of $N$. meningitidis cultured from the cervix of a patient.

\section{Introduction}

Neisseria species other than Neisseria gonorrhoeae have been found to cause symptomatic infections of the genitourinary tract and the anal canal. A report of an increase in incidence of isolation as well as a brief review of the literature on Neisseria meningitidis infections of these sites has been recently published (Givan et al., 1977). In the present study we isolated 285 non-gonococcal species of Neisseria. Among these there were four $N$. meningitidis isolates and five Neisseria catarrhalis isolates from genitourinary or rectal sites. Our findings for $N$. meningitidis differ from those previously reported-in particular, the number of male patients in the series from whom the organism was isolated, overt signs of infection, autoinoculation from the nasopharynx as a source of the organism. The possibility of inhibitory substances produced by $N$. meningitidis preventing infection by $N$. gonorrhoeae in one patient is discussed.

\section{Materials and methods}

PATIENTS

The patients in this study attended the Department of Venereology, Edinburgh Royal Infirmary between 1 July 1976 and 30 June 1977. During this

Address for reprints: C. Blackwell, Department of Bacteriology, University of Edinburgh, Medical School, Teviot Place, Edinburgh EH8 9AG

Received for publication 22 July 1977 period there were approximately 2000 women and 2500 men.

In men, if a urethral discharge was present, or if there was presumptive evidence of contact with gonorrhoea, a Gram-stained film of urethral discharge was examined microscopically and material inoculated directly on to culture plates. In the case of homosexuals, material from the urethra, rectum and pharynx was cultured routinely, and if negative on the first occasion, repeated twice at weekly intervals.

In women, Gram-stained films of material from the urethra and cervix, and cultures from the urethra, cervix, rectum and, if indicated, from the pharynx were taken. Generally, if the first cultures were negative for $N$. gonorrhoeae, these were repeated twice at weekly intervals.

CULTURE AND IDENTIFICATION

During the first six months of the study cultures were made on Thayer-Martin medium containing the antibiotics vancomycin $(4.0 \mu \mathrm{g} / \mathrm{ml})$, colistin $(6.0 \mu \mathrm{g} / \mathrm{ml})$, and nystatin $(10.0 \mu \mathrm{g} / \mathrm{ml})$. During 1977 , cultures were made on modified New York City Medium (Young, 1978) containing the antibiotics lincomycin $(1.0 \mu \mathrm{g} / \mathrm{ml})$, colistin $(6.0 \mu \mathrm{g} / \mathrm{ml})$, amphotericin $B(1.0 \mu \mathrm{g} / \mathrm{ml})$, and trimethoprim lactate $(6 \cdot 5 \mu \mathrm{g} / \mathrm{ml})$.

Cultures were incubated at $36^{\circ} \mathrm{C}$ in a carbon dioxide enriched $(10 \%)$ atmosphere. After incubation for 24 hours, plates were examined and any suspect colonies tested by the oxidase reaction and Gram's stain. Oxidase positive Gram-negative diplococci 
were identified by the rapid carbohydrate utilisation test and direct immunofluorescence using Difco fluorescein-labelled anti-gonococcal conjugate as described by Young et al. (1976). Negative cultures were re-examined after 48 hours of incubation.

Demonstration of the inhibitory substance from $N$. meningitidis was by means of the soft agar method for bacteriocin production (Fredericq, 1957).

\section{Results}

During the 12-month period from 1 July 1976 to 30 June 1977 approximately 4500 patients attended the Department of Venereology, Royal Infirmary, Edinburgh. There were 350 specimens of $N$. gonorrhoeae isolated from the 2000 female patients and 480 specimens isolated from the 2500 male patients.

The Neisseria isolates, other than N. gonorrhoeae, from these patients are summarised in Table 1.

Table 1 Isolation of Neisseria species other than $\mathrm{N}$ gonorrhoeae over a 12-month period

\begin{tabular}{lrrll}
\hline \multirow{2}{*}{ Neisseria } & $\begin{array}{l}\text { Total no. } \\
\text { of isolates }\end{array}$ & Throat & $\begin{array}{l}\text { Genito- } \\
\text { urinary tract }\end{array}$ & $\begin{array}{l}\text { Anal } \\
\text { canal }\end{array}$ \\
\cline { 3 - 5 } & 250 & 246 & 2 & 2 \\
N. meningitidis & 250 & 23 & 0 & 0 \\
N. lactamica & 23 & 2 & 1 & 4 \\
N. catarrhalis & 7 & 3 & 0 & 0 \\
N. flava & 3 & 2 & 0 & 0 \\
N. perflava & 2 & & & \\
\hline
\end{tabular}

$N$. meningitidis was the species most often isolated (250); among these there were two from the anal canal and two from the genitourinary tract. We found $N$. lactamica (23), $N$. flava (3), and $N$. perflava (2) only in throat cultures. Of the seven $N$. catarrhalis, two were from throat cultures, one was from the genitourinary tract, and four from the anal canal.

There were four patients from whom $N$. meningi- tidis was isolated. The information available for each patient is summarised in Table 2. The only additional systemic complaints from Patient 1 were a loss in weight of $7 \mathrm{lb}(3.175 \mathrm{~kg})$, dysuria, and frequency of micturition. $N$. gonorrhoeae were not isolated at this time nor on the three subsequent sets of cultures. She had had a regular consort for the past two years and the only previous history of a sexually transmitted disease (STD) was warts in 1975 and genital candidosis in 1974. A second rectal isolate was from Patient 2 who had attended the clinic four months previously, at which time $N$. meningitidis was isolated from the throat. The only systemic complaint was eczema on face, trunk, and limbs and there was no previous history of STD. Patient 3, a bisexual male with no previous history of STD, had attended the clinic two months earlier. At that time $N$. meningitidis was isolated from the throat but no Neisseria had been found in cultures of rectum or urethra.

The only patient in the series from which we isolated $N$. meningitidis and, later, $N$. gonorrhoeae was Patient 4. She was seen in the clinic as a gonorrhoea contact; her husband had become infected from an extramarital consort. Microscopical examination of material from the cervix revealed large numbers of polymorphonuclear leucocytes with intracellular Gram-negative diplococci. She was treated with co-trimoxazole as there was a possibility of penicillin allergy. The culture of cervical material grew only $N$. meningitidis and no gonococci were isolated from any other site. A month later gonococci were cultured from the cervix and she was treated with another course of co-trimoxazole. Disc sensitivity tests revealed both the previously isolated meningococcus and the gonococcus to be sensitive to trimethoprimsulphamethoxazole (disc containing 23.5 $\mu \mathrm{g}$ sulphamethoxazole, $1.5 \mu \mathrm{g}$ trimethoprim). At the time it was serotyped, the meningococcus was

Table 2 Isolation of $\mathrm{N}$. meningitidis

\begin{tabular}{|c|c|c|c|c|c|c|}
\hline Patient & $\operatorname{Sex}$ & Site of isolation & Throat culture & Serotype & $\begin{array}{l}\text { Reason for } \\
\text { attending STD clinic }\end{array}$ & Diagnosis, treatment, and follow-up \\
\hline 1 & $\mathbf{F}$ & Rectum & - & B & Warts & $\begin{array}{l}\text { Cautery and liquid nitrogen for warts; } \\
3 \text { subsequent sets of cultures negative }\end{array}$ \\
\hline 2 & $\mathbf{F}$ & Rectum & Not done & B & $\begin{array}{l}\text { Frequency of } \\
\text { micturition; warts }\end{array}$ & $\begin{array}{l}\text { Podophyllin and cautery for warts; no } \\
\text { follow-up cultures subsequent to isolation } \\
\text { of } N \text {. meningitidis from anal canal }\end{array}$ \\
\hline 3 & $\mathbf{M}$ & Urethra & Not done & $\mathbf{N T}$ & Syphilis contact & No treatment; no follow-up cultures \\
\hline 4 & $\mathbf{F}$ & Cervix & - & NT & $\begin{array}{l}\text { Gonorrhoea } \\
\text { contact }\end{array}$ & $\begin{array}{l}\text { Asymptomatic gonorrhoea (only } N \text {. } \\
\text { meningitidis cultured); co-trimoxazole } 5 \\
\text { days; } 2 \text { subsequent sets of cultures } \\
\text { negative; } N \text {. gonorrhoeae cultured } 3 \text { times } \\
\text { during next } 3 \text { months }\end{array}$ \\
\hline
\end{tabular}

$-=$ no Neisseria isolated

$\mathrm{NT}=$ non sero-typable $N$. meningitidis 
further tested and found to grow on $0.1 \mu \mathrm{g} / \mathrm{ml}$ sulfadiazine but not on $1 \mu \mathrm{g} / \mathrm{ml}$. She denied any sexual contact with her husband during this period. The soft agar method for demonstrating sensitivity to bacteriocins showed the meningococcus elaborated a substance which greatly inhibited the gonococci from Patient 4 and several other $N$. gonorrhoeae isolates from different sources. It was later discovered that the gonococcal infection was probably not a treatment failure or inhibition by the meningococcal substance but infection from an extramarital contact. There was no systemic upset in Patient 4, but her extramarital contact and his wife developed systemic infections and arthritis.

There were four female patients from whom $N$. catarrhalis was isolated (Table 3). None of these had any systemic upset and no gonococci were cultured at follow-up visits.

\section{Discussion}

Givan et al. (1977) have reviewed case reports of isolation of $N$. meningitidis from the genitourinary tract and anal canal. Our data from the past 12 months provide a somewhat different view. These differences may be owing to routine culturing of urethra, cervix, and rectum of all female patients. Previous reports mention only cervical cultures of women, with additional blood or joint cultures from two systemic infections (Keys et al., 1971; Morgan et al., 1976). Other series contain a predominance of men, 58 of the 84 patients in the literature; in our study three of the four patients from whom we isolated $N$. meningitidis and all of the patients from whom we isolated $N$. catarrhalis were women.

There were no overt symptoms of these meningococci causing infection in any of the patients, again in contrast to the findings of others. The main reason for attending the Department of Venereology was genital warts (four out of eight patients). Three were either syphilis or gonorrhoea contacts, and the fourth was referred for follow-up of a gonococcal infection. There was no evidence of rectal infections with $N$. meningitidis associated with systemic infection. Currently there is no evidence for treating rectal carriers, but follow-up cultures to monitor any spread to the urethra or the cervix may be of value.

It has been suggested (Givan et al., 1977) that there may be autoinoculation of these areas from the nasopharynx. In their seriès all six of the rectal carriers from whom there were throat cultures had meningococcus in the pharynx. In our series, none of the patients from whom throat cultures were taken was carrying $N$. meningitidis in the nasopharynx. It is also interesting to note that meningococci were not isolated from either contact of Patient 4.

The significance of the inhibitory effect of $N$. meningitidis from Patient 4 on $N$. gonorrhoeae is difficult to assess. A similar instance of apparent protection has been reported for a male patient with asymptomatic meningococcal urethritis (Volk and Kraus, 1973). The role of this inhibitory effect in preventing infection from her initial exposure is not clear. The meningococcus would probably have been eliminated by the first co-trimoxazole treatment before her second exposure and subsequent infection with $N$. gonorrhoeae.

These observations underline the necessity of correct and full identification of all neisserial isolates, even when a selective medium is used. These non-gonococcal Neisseria represent approximately $2 \%$ of the anogenital isolates in women and $0.2 \%$ of those from men.

This work is part of a project supported by a grant from the Scottish Home and Health Department, Medical Research Committee.

Serotyping of meningococci and sulphadiazine sensitivity testing was kindly carried out by $\mathrm{Dr}$ R. J. Fallon, Department of Laboratory Medicine, Ruchill Hospital, Glasgow.

Table 3 Isolation of $\mathrm{N}$. catarrhalis

\begin{tabular}{|c|c|c|c|c|c|}
\hline Patient & $\operatorname{Sex}$ & Site of isolation & Throat culture & $\begin{array}{l}\text { Reason for attending } \\
\text { STD clinic }\end{array}$ & Diagnosis, treatment, and follow-up \\
\hline 5 & $\mathbf{F}$ & Rectum & - & Gonorrhoea contact & $\begin{array}{l}\text { No treatment; } 3 \text { subsequent sets of } \\
\text { cultures negative }\end{array}$ \\
\hline 6 & $\mathbf{F}$ & Rectum & Not done & Warts & $\begin{array}{l}\text { Podophyllin for warts; } 3 \text { subsequent sets } \\
\text { of cultures negative }\end{array}$ \\
\hline 7 & $\mathbf{F}$ & Rectum & Not done & $\begin{array}{l}\text { Follow-up treatment for } \\
\text { gonorrhoea }\end{array}$ & $\begin{array}{l}\text { Non-gonococcal vaginal discharge; } \\
\text { nystatin for discharge }\end{array}$ \\
\hline 8 & $\mathbf{F}$ & $\begin{array}{l}\text { Urethra } \\
\text { Rectum }\end{array}$ & Not done & Warts & $\begin{array}{l}\text { Podophyllin for warts; } 4 \text { subsequent sets } \\
\text { of cultures negative }\end{array}$ \\
\hline
\end{tabular}


References

Fredericq, P. (1957). Colicins. Annual Review of Microbiology, 11, 7-22.

Givan, K. F., Thomas, B. W., and Johnston, A. G. (1977). Isolation of Neisseria meningitidis from the urethra, cervix, and anal canal: Further observations. British Journal of Venereal Diseases, 53, 109-112.

Keys, T. F., Hecht, R. H., and Chow, A. W. (1971). Endocervical Neisseria meningitidis with meningococcemia. New England Journal of Medicine, 285, 505-506.

Morgan, J. R., Fedder, J., and Smith, J. A. (1976). Postpartum infection with meningococcemia. Canadian Medical Association Journal, 114, 294.
Volk, J., and Kraus, S. J. (1973). Asymptomatic meningococcal urethritis. Possible protective value against gonococcal infection by bacteriocin production. British Journal of Venereal Diseases, 49, 511-512.

Young, H. (1978). Cultural diagnosis of gonorrhoea with modified New York City (MNYC) medium. British Journal of Venereal Diseases, 54, 36-40.

Young, H., Paterson, I. C., and McDonald, D. R. (1976). Rapid carbohydrate utilisation test for the identification of Neisseria gonorrhoeae. British Journal of Venereal Diseases, 52, 172-175. 